\title{
MINIMAL COMPACTIFICATIONS AND THEIR ASSOCIATED FUNCTION SPACES
}

\author{
GARY D. FAULKNER
}

(Communicated by James E. West)

\begin{abstract}
This paper investigates the association between compactifications of a space which are minimal with respect to the extension of families of continuous functions and their associated subalgebras of $C^{*}(X)$.
\end{abstract}

\section{INTRODUCTION}

Let $X$ be a locally compact, noncompact Hausdorff space. With each compactification $\alpha X$ of $X$ we can associate a subalgebra of $C^{*}(X)$, the collection of all bounded continuous real valued functions on $X$, as follows: $C_{\alpha}(X)$ is the collection of all function in $C^{*}(X)$ which have continuous extensions to $\alpha X$. It is well known that this association is a lattice isomorphism between the compactifications of $X$ and the family of algebras so obtained. For any collection $\mathscr{G}$ of continuous functions $f_{\gamma}: X \rightarrow K_{\gamma}$, where $K_{\gamma}$ is a compact Hausdorff space, there is a smallest compactification to which the entire collection $\mathscr{G}$ extends [9]. This minimal compactification can be realized by adjoining the singular set of an appropriate function to $X$. Singular sets and their relation to compactifications have been studied extensively [1], [2], [3], [5], [6]. In the event that a single real valued continuous function is being extended we can view the resulting compactification as fundamental. This paper is concerned with these minimal compactifications and their associated function algebras.

\section{NotATION AND DEFINITIONS}

In what follows all spaces will be locally compact Hausdorff. The real numbers will be denoted by $\mathbf{R}$. For a space $X, C^{*}(X)$ will denote the algebra of bounded continuous real valued functions on $X$. If $\alpha X$ is a compactification of $X$, then $C_{\alpha}(X)$ will denote the subalgebra of $C^{*}(X)$ consisting of functions which have continuous extensions to $\alpha X$. $\beta X$ and $\omega X$ will denote the StoneCech and the Alexandroff one-point compactifications of $X$ respectively. Note that $C_{\beta}(X)$ is the same as $C^{*}(X)$, while $C_{\omega}(X)$ consists of those functions

Received by the editors October 13, 1987 and, in revised form, April 16, 1989.

1980 Mathematics Subject Classification (1985 Revision). Primary 54D35, 54D40, 54C10.

Key words and phrases. Compactification, singular set, algebra of functions. 
which are constant at infinity. For our purposes we will say that two continuous real valued functions are equivalent if their difference is an element of $C_{\omega}(X)$. If $f$ and $g$ are equivalent we will denote this by $f \cong g$. If $\mathscr{F}$ and $\mathscr{G}$ are two collections of functions we will say that $\mathscr{F} \cong \mathscr{G}$ provided each function in $\mathscr{F}$ is equivalent to a function in $\mathscr{G}$ and conversely. If $\mathscr{G} \subseteq C^{*}(X)$ then $e_{\mathscr{G}}$ will denote the product mapping. In other words, if $\mathscr{G}=\left\{f_{\gamma}\right\}$ where $f_{\gamma}: X \rightarrow I_{\gamma}$ ( $I_{\gamma}$ is a closed bounded subinterval of $\mathbf{R}$ ), then $e_{\mathscr{G}}(x)=\left\langle f_{\gamma}(x)\right\rangle \in \prod_{\gamma} I_{\gamma}$. If $\mathscr{G}$ separates points from closed sets, then $e_{\mathscr{G}}$ is an embedding. If $f \in C_{\alpha}(X)$, then $f^{\alpha}$ will denote its extension to $\alpha X$. If $\mathscr{G} \subseteq C_{\alpha}(X)$, then $\mathscr{G}^{\alpha}$ will denote the family of extensions of functions in $\mathscr{G}$ to $\alpha X$. For $\mathscr{G} \subseteq C^{*}(X),\langle\mathscr{G}\rangle$ will denote the algebra generated by $\mathscr{G}$. If $K$ is compact and $f: X \rightarrow K$ is continuous, then the singular set of $f, \mathscr{S}(f)$ is defined by

$\mathscr{S}(f)=\left\{p \in K \mid\right.$ for each open $U$ with $p \in U \subseteq K, \overline{f^{-1}(U)}$ is not compact $\}$.

If $\mathscr{G} \subseteq C^{*}(X)$, then $\omega_{\mathscr{G}} X$ will denote the smallest compactification to which the collection $\mathscr{G}$ extends. In the case that $\mathscr{G}=\{f\}$, this is written as $\omega_{f} X$. For any set $A$, the cardinality of $A$ will be denoted by $|A|$. If $\alpha X$ and $\gamma X$ are two compactifications with $\alpha X \leq \gamma X$ then the projection from $\gamma X$ to $\alpha X$ will be denoted by $\pi_{\gamma \alpha}$. The projection onto the $\gamma$ th factor of a product will be denoted by $\pi_{\gamma}$. All other notation is standard. Generally the notation here conforms to that of [4].

\section{RESUlts}

Let $f \in C^{*}(X)$. In [7] it is shown that the extension of $f$ to $\omega_{f} X$ is one-toone on the remainder. Furthermore $f^{\omega}\left(\omega_{f} X \backslash X\right)=\mathscr{S}(f)$ [5], so that $\mathscr{S}(f)$ is homeomorphic to $\omega_{f} X \backslash X$. Furthermore, $f$ extends to $\omega_{f} X$ as the identity on the remainder, which can be viewed as a subset of $[0,1]$. It seems that these compactifications are in some sense the simplest compactifications in the lattice. As we will see, they play a fundamental role in the structure of general compactifications.

If $\alpha X$ is an arbitrary compactification of $X$ and $f \in C^{*}(X)$, then there is a smallest compactification greater than $\alpha X$ to which $f$ extends. This compactification is $\gamma X=\sup \left\{\alpha X, \omega_{f} X\right\}$. The case covered in [7] corresponds to the case where $\alpha X=\omega X$. Although the extension of $f$ to $\gamma X$ need not be one-to-one on $\gamma X \backslash X$, it is one-to-one on each of the fibers, $\pi_{\gamma \alpha}^{-1}(p)$. We will make use of this fact later.

We begin with a relative of the Stone-Weierstrass Theorem.

Lemma 1. Let $\mathscr{G}$ be a subalgebra of $C_{\alpha}(X)$. Suppose that $C_{\omega}(X) \subseteq \mathscr{G}$ and $\mathscr{G}^{\alpha}$ separates points of $\alpha X \backslash X$; then $\overline{\mathscr{G}}=C_{\alpha}(X)$.

Proof. Note that $\mathscr{G}^{\alpha}$ contains all of the constant functions on $\alpha X$. Let $p \in \alpha X$ and $q \in X$. Let $V$ be a neighborhood with compact closure such that $q \in V \subseteq$ $X$, and $p \notin V$. Choose a Urysohn function $g$ on $\alpha X$ which is supported in 
$V$ and for which $g(q)=1$. Clearly $\left.g\right|_{X} \in C_{\omega}(X) \subseteq \mathscr{G}$. In addition $g=\left(\left.g\right|_{X}\right)^{\alpha}$ separates $p$ and $q$. By hypothesis $\mathscr{G}$ separates points in $\alpha X \backslash X$ so that the algebra $\mathscr{G}^{\alpha}$ contains the constant functions and separates points of $\alpha X$. By the Stone-Weierstrass Theorem $\overline{\mathscr{G}}^{\alpha}=C(\alpha X)$. Now let $j: C(\alpha X) \rightarrow C_{\alpha}(X)$ be defined by $j(f)=\left.f\right|_{X}$. Since $j$ is an isometric isomorphism, $j\left(\mathscr{G}^{\alpha}\right)=\mathscr{G}$ is dense in $C_{\alpha}(X)$.

We observe that the minimum compactification to which a collection $\mathscr{G} \subseteq$ $C^{*}(X)$ extends is the same as the minimum compactification to which the product mapping $e_{\mathscr{G}}$ extends. To see this suppose that each $f \in \mathscr{G}$ extends to $\alpha X$. In this case $e_{\mathscr{G}}$ also extends via $\left\langle f_{\gamma}^{\alpha}\right\rangle$. Conversely, if $e_{\mathscr{G}}$ extends to $\alpha X$, then $\pi_{\gamma} \circ e_{\mathscr{G}}^{\alpha}$ is an extension of $f_{\gamma}$. Thus the collection of compactifications to which each extends is the same, from which it follows that the minimum is also the same.

Theorem 1. Let $\mathscr{G} \subseteq C^{*}(X)$ separate points from closed sets in $X$. Let eX be the compactification obtained from the embedding $e_{\mathscr{G}}$, that is $e X=\overline{e_{\mathscr{G}}(X)} \subseteq$ $\prod_{f \in \mathscr{G}} I_{f}$. Then $C_{e}(X)=\overline{\left\langle C_{\omega}(X) \cup \mathscr{G}\right\rangle}$.

Proof. Clearly $e_{\mathscr{G}}$ has an extension to $e X$ which is one-to-one on $e X \backslash X$. In particular $e X$ is the smallest compactification to which the collection $\mathscr{G}$ extends. Thus the functions in $\mathscr{G}$ must separate points in $e X \backslash X$. Since each function in $\mathscr{G}$ extends to $e X$ we have $\left\langle C_{\omega}(X) \cup \mathscr{G}\right\rangle \subseteq C_{e}(X)$. By Lemma 1 the theorem follows.

Corollary 1. Let $\mathscr{G} \subseteq C^{*}(X)$. Then whether or not $\mathscr{G}$ separates points from closed sets in $X$,

$$
C_{\omega_{\mathscr{G}}}(X)=\overline{\left\langle C_{\omega}(X) \cup \mathscr{G}\right\rangle}
$$

Proof. Let $\mathscr{F}=C_{\omega}(X) \cup \mathscr{G}$. Then $\mathscr{F}$ separates points from closed sets in $X$ and $\left\langle C_{\omega}(X) \cup \mathscr{G}\right\rangle=\left\langle C_{\omega}(X) \cup \mathscr{F}\right\rangle$. Since each function in $C_{\omega}(X)$ extends to every compactification, $\omega_{\mathscr{G}} X=\omega_{\mathscr{F}} X$. Thus

$$
C_{\omega_{\mathscr{G}}}(X)=C_{e_{\mathscr{F}}}(X)=\overline{\left\langle C_{\omega}(X) \cup \mathscr{F}\right\rangle}=\overline{\left\langle C_{\omega}(X) \cup \mathscr{G}\right\rangle} \text {. }
$$

Corollary 2. In order that a closed subalgebra $\mathscr{F}$ of $C^{*}(X)$ be $C_{\alpha}(X)$ for some $\alpha X$ it is necessary and sufficient that $\mathscr{F}$ contain $C_{\omega}(X)$.

Proof. Clearly if $\mathscr{F}=C_{\alpha}(X)$ for some $\alpha X$ then $C_{\omega}(X) \subseteq \mathscr{F}$. Conversely if $C_{\omega}(X) \subseteq \mathscr{F}$, then $\mathscr{F}=\overline{\left\langle C_{\omega}(X) \cup \mathscr{F}\right\rangle}=C_{\omega_{\mathscr{F}}}(X)$.

Corollary 3. If $\mathscr{F}$ and $\mathscr{G}$ are two equivalent subsets of $C^{*}(X)$, then $e_{\mathscr{G}}=e_{\mathscr{F}}$ (or equivalently $\omega_{\mathscr{G}} X=\omega_{\mathscr{F}} X$ ).

Proof. If $\mathscr{F}$ and $\mathscr{G}$ are equivalent then $\left\langle C_{\omega}(X) \cup \mathscr{G}\right\rangle=\left\langle C_{\omega}(X) \cup \mathscr{F}\right\rangle$.

Example 1. The converse of Corollary 3 is not true even in the simplest case. Let $X$ be the disjoint union of two copies, $\Omega_{1}$ and $\Omega_{2}$, of the ordinals less than the first uncountable ordinal. In this case $\beta X$ is the two point compactification of $X$. Let $f_{k}$ be the characteristic function of $\Omega_{k}(k=1,2)$. Clearly the two 
functions are not equivalent, but each satisfies $\beta X=\omega_{f_{k}} X$. We note that each is a polynomial in the other with coefficients from $C_{\omega}(X)$. This is of course more general than being equivalent.

Corollary 4. The lattice of compactifications of a space $X$ is isomorphic to the lattice of closed subalgebras of $C^{*}(X)$ containing $C_{\omega}(X)$.

Proof. This follows easily from Corollary 2 .

Lemma 2. Let $\alpha_{t} X$ be a family of compactification of $X$ and let $\alpha X=\sup _{t} \alpha_{t} X$; then $C_{\alpha}(X)=\overline{\left\langle\bigcup_{l} C_{\alpha_{l}}(X)\right\rangle}$.

Proof. By Corollary 2, $\overline{\left\langle\bigcup_{l} C_{\alpha_{t}}(X)\right\rangle}$ is the function space associated with a compactification. Thus the lemma follows from the fact that the lattice of compactifications and the lattice of associated function spaces are isomorphic.

Theorem 2. Let $\mathscr{G} \subseteq C^{*}(X)$ separate points from closed sets in $X$; then the following holds:

$$
\sup _{f \in \mathscr{G}} \omega_{f} X=\omega_{\mathscr{G}} X=e_{\mathscr{G}} X .
$$

Proof. Let $\gamma X=\sup _{f \in \mathscr{G}} \omega_{f} X$. Then clearly $\gamma X \leq \omega_{\mathscr{G}} X \leq e_{\mathscr{G}} X$. Also by Theorem 1 and Lemma 2 it follows that $C_{e_{\mathscr{S}}}(X)=\overline{\left\langle C_{\omega}(X) \cup \mathscr{G}\right\rangle} \subseteq C_{\gamma}(X)$. Hence $e_{\mathscr{G}} X \leq \sup _{f \in \mathscr{G}} \omega_{f} X$.

From the above it follows that $\beta X=\sup _{f \in \mathscr{G}} \omega_{f} X$ for appropriate collections $\mathscr{G} \subseteq C^{*}(X)$. In fact every compactification of $X$ satisfies a similar relation. For the case of $\beta X, \mathscr{G}$ need not be all of $C^{*}(X)$. In particular $\mathscr{G}$ need not contain equivalent functions. In the case that $|\beta X \backslash X| \leq \aleph_{0}, \mathscr{G}$ may consist of a singleton [7]. Since $\left(\sup \omega_{f} X\right) \backslash X$ is homeomorphic to a subset of $\prod_{f \in \mathscr{G}} \mathscr{S}(f)$, it follows from cardinality considerations alone that if $\beta X=\sup _{f \in \mathscr{G}} \omega_{f} X$, then $|\mathscr{G}|>|\mathbf{R}|$ whenever $X$ is real compact. From this it follows that there are at least $|\mathbf{R}|$ nonequivalent functions in $C^{*}(X)$ for real compact $X$.

It also follows from Theorem 2 that for spaces such as $n$-dimensional Euclidean space $(n \geq 2)$ every compactification is a supremum of compactifications having closed intervals as remainders. This it true since for these spaces each remainder is connected. In this case, for each $f \in C^{*}(X)$, either $\mathscr{S}(f)$ is a single point or $\mathscr{S}(f) \cong[0,1]$.

In what follows we will be interested in the algebra associated with $\omega_{f} X$. In particular, when is it $\left\langle C_{\omega}(X) \cup\{f\}\right\rangle$ without closure. In this case $C_{\omega_{f}}(X)$ is a singly generated module over $C_{\omega}(X)$. Let $\alpha X \leq \gamma X$. We will say that $\pi_{\gamma / \alpha}$ is finite if

$$
\left|\bigcup\left\{\pi_{\gamma / x}^{-1}(p):\left|\pi_{\gamma / x}^{-1}(p)\right|>1\right\}\right|<\infty .
$$

This is to say that the projection has only finitely many point inverses which do not consist of a singleton and each of these consists of only finitely many points. 
Theorem 3. Let $\alpha X \leq \gamma X$ and suppose that $\pi_{\gamma \alpha}$ is finite; then there exists an $f \in C^{*}(X)$ such that

$$
C_{\gamma}(X)=\left\langle C_{\alpha}(X) \cup\{f\}\right\rangle \text {. }
$$

Proof. Let $\left\{p_{1}, \ldots, p_{n}\right\}=\bigcup\left\{\pi_{\gamma \alpha}^{-1}(p):\left|\pi_{\gamma \alpha}^{-1}(p)\right|>1\right\}$. Let $\left\{U_{k}\right\}_{k=1}^{n}$ be open subsets of $\gamma K$, with disjoint closures, such that $p_{k} \in U_{k}$. By Urysohn's Lemma there is a function $f \in C_{\gamma}(X)$ such that $f\left(\overline{U_{k}}\right) \equiv k$.

Note that $C_{\alpha}(X)$ consists of those $g \in C_{\gamma}(X)$ such that $g^{\gamma}$ is constant on sets of the form $\pi_{\gamma \alpha}^{-1}(p)$. Let

$$
f_{j}=\frac{\prod_{j \neq l}(f-l)}{\prod_{j \neq l}(j-l)} .
$$

Clearly $f_{k} \in\left\langle C_{\alpha}(X) \cup\{f\}\right\rangle$ since each is a polynomial in $f$ with coefficients in $C_{\alpha}(X)$. Furthermore

$$
f_{k}\left(U_{j}\right)= \begin{cases}1 & \text { if } j=k \\ 0 & \text { if } j \neq k\end{cases}
$$

Let $\hat{f}_{k}$ denote the restriction of $f_{k}$ to $X$. Now let $g \in C_{\gamma}(X)$. Define $g_{j}$ as follows:

$$
g_{j}=\left(g-g^{\gamma}\left(p_{j}\right)\right) \hat{f}_{j} .
$$

Since $g_{j}^{\gamma}(x)=0$ for $x \in\left\{\pi_{\gamma \alpha}^{-1}(p):\left|\pi_{\gamma_{\alpha} \alpha}^{-1}(p)\right|<1\right\}$, we have $g_{j} \in C_{\alpha}(X)$. Let $h=\sum g_{j}$. It also follows that $h \in C_{\alpha}(X)$. Finally let

$$
s=\sum\left(h+g\left(p_{j}\right)\right) \hat{f}_{j} .
$$

Routine computation shows that $s-g \in C_{\alpha}(X)$ since $s^{\gamma}\left(p_{j}\right)=g^{\gamma}\left(p_{j}\right)$. Also $s \in\left\langle C_{\alpha}(X) \cup\{f\}\right\rangle$. Thus $g=s-(s-g) \in\left\langle C_{\alpha}(X) \cup\{f\}\right\rangle$.

A partial converse of this theorem is true. I do not know if the complete converse is true.

Theorem 4. Let $C_{\gamma}(X)=\left\langle C_{\alpha}(X) \cup\{f\}\right\rangle$; then $\left|\pi_{\gamma_{\alpha}}^{-1}(p)\right|<\infty$ for each $p \in \alpha X$. Proof. First note that $\gamma K$ is the smallest compactification larger than $\alpha X$ to which $f$ extends, so that $f^{\gamma}$ is one-to-one on $\pi_{\gamma \alpha}^{-1}(p)$ for each $p \in \alpha X \backslash X$. Let $p \in \alpha X \backslash X$ and let $K=f^{\gamma}\left(\pi_{\gamma_{\alpha}}^{-1}(p)\right) \subseteq \omega_{f} X \backslash X \subseteq \mathbf{R}$. Let $g=\sin (f)$. Now $g \in C_{\omega}(X) \subseteq C \gamma(X)$ so that $g$ is a polynomial in $f$ with coefficients from $C_{\alpha}(X)$. Let $q$ denote this polynomial. On $\pi_{\gamma \alpha}^{-1}(p)$, the coefficients of $g^{\gamma}=q^{\gamma}$ are constant. Suppose $g^{\gamma}$ agrees with $a_{n}\left(f^{\gamma}\right)^{n}+\cdots+a_{1} f^{\prime}+a_{0}$ on $\pi_{\gamma^{\prime}(\alpha}^{-1}(p)$. Now $f$ extends to $\omega_{f} X$ as the identity on the remainder. This means that $\sin x$ and $a_{n} x^{n}+\cdots+a_{1} x+a_{0}$ agree on $K \subseteq \mathbf{R}$. This can happen only if $|K|<\infty$. Since $f^{\gamma}$ is one-to-one from $\pi_{j \alpha}^{-1}(p)$ into $K$, the theorem follows. 
Theorem 5. Let $\alpha X$ have a finite remainder and let $\alpha X \leq \gamma X$. Then $\gamma X$ has a finite remainder if and only if $C_{\gamma}(X)=\left\langle C_{\alpha}(X) \cup\{f\}\right\rangle$.

Proof. This follows easily from Theorems 3 and 4.

\section{ACKNOWLEDGMENTS}

I would like to thank my friends Richard Chandler and Michael Singer for their very helpful conservations during the preparation of this paper.

\section{REFERENCES}

1. G. L. Cain, Jr., Compact and related mappings, Duke Math. J. 33 (1966), 639-645.

2. __ Mappings with prescribed singular sets, Nieuw. Arch. Wisk. 17 (3) (1969), 200-203.

3. G. L. Cain, Jr., R. E. Chandler and G. D. Faulkner, Singular sets and remainders, Trans. Amer. Math. Soc. 268 (1961), 161-171.

4. R. E. Chandler, Hausdorff compactifications, Marcel Dekker, New York, (1976).

5. R. E. Chandler and G. D. Faulkner, Singular compactifications: The order structure, Proc. Amer. Math. Soc. 100 (1987), 377-382.

6. R. E. Chandler, G. D. Faulkner, J. P. Gugliemi and M. Memory, Generalizing the AlexandroffUrysohn double circumference construction, Proc. Amer. Math. Soc. 83 (1981), 606-608.

7. R. E. Chandler and R. Gellar, The compactifications to which an element of $C^{*}(X)$ extends, Proc Amer. Math. Soc. 38 (1973), 637-639.

8. R. E. Chandler and F. C. Tzung, Remainders in Hausdorff compactifications, Proc. Amer. Math. Soc. 70 (1978), 196-202.

9. P. A. Loeb, A minimal compactification for extending continuous functions, Proc. Amer. Math. Soc. 18 (1969), 282-283.

North Carolina State University, Mathematics Department, Raleigh, North CAROLINA 27695 